\title{
Downlink compressive channel estimation with support diagnosis in FDD massive MIMO
}

\author{
Wei Lu ${ }^{1 *} \mathbb{D}$, Yongliang Wang ${ }^{1}$, Qiqing Fang ${ }^{1}$ and Shixin Peng ${ }^{2}$
}

\begin{abstract}
Downlink channel state information (CSI) is critical in a frequency division duplexing (FDD) massive multiple-input multiple-output (MIMO) system. We exploit the reciprocity between uplink and downlink channels in angular domain and diagnose the supports of downlink channel from the estimated uplink channel. While the basis mismatch effects will damage the sparsity level and the path angle deviations between uplink and downlink transmission paths will induce differences in channel supports, a downlink support diagnosis algorithm based on the DBSCAN (density-based spatial clustering of applications with noise) which is widely used in machine learning is presented. With the diagnosed supports of downlink channel in angular domain, a weighted subspace pursuit (SP) channel estimation algorithm for FDD massive MIMO is proposed. The restricted isometry property (RIP)-based performance analysis for the weighted SP algorithm is given out. Both the analysis and the simulation results show that the proposed downlink channel estimation with diagnosed supports is superior to the standard iteratively reweighted least squares (IRLS) and SP without channel priori or with the assumption of the common supports for uplink and downlink channels in angular domain.
\end{abstract}

Keywords: FDD, Massive MIMO, Support diagnosis, Compressive channel estimation, Weighted subspace pursuit

\section{Introduction}

Spectrum and radio resources in the forthcoming new communication systems are valuable for efficient transmission. Cognitive radio technologies are used for spectrum sensing to reduce the spectrum idle rate, while compressed sensing technologies are applied to improve utilization efficiency of radio resource $[1,2]$. Compressed sensing (CS) can be applied into a massive multiple-input multiple-output (MIMO) system for efficient transmission. It is crucial to have accurate channel state information (CSI) at transmitter for downlink beamforming in massive MIMO. In a time division duplexing (TDD) massive MIMO system, downlink CSI can be obtained by exploiting the channel reciprocity from the uplink channel. In frequency division duplexing (FDD) massive MIMO system, downlink CSI feedback is challenging since the training and feedback overhead are proportional to the antenna number at the base station (BS) if the

\footnotetext{
* Correspondence: Ivweiwhut@aliyun.com

${ }^{1}$ Air Force Early Warning Academy, Wuhan, China

Full list of author information is available at the end of the article
}

feedback scheme in LTE is adopted [3]. Motivated by the framework of CS and the sparsity of massive MIMO channel in angular domain also known as spatial domain, applications of CS to massive MIMO channel estimation and feedback have been intensively studied.

In the compressive channel estimation, it can benefit from the CS technology to reduce the training and feedback overhead and profit from the priori knowledge about the channel support to improve the estimation performance further. The users feed the compressed training measurements back to the BS to reduce feedback overhead, and orthogonal matching pursuit (OMP) is used for downlink CSI recovery in [4]. In [5], the modified basis pursuit (MBP) is proposed by utilizing the partial priori signal support information to improve the recovery performance. In [6], Bayesian estimation of sparse massive MIMO channel is developed in which neighboring antennas share among each other their information about the channel support. A weighted CS-based uplink channel estimation is considered in TDD massive MIMO, and the previous estimated channel is used for generating weights for CS recovery in 
[7]. In [8], the previous channel support is used for the initialization of the estimated support for subspace pursuit. In [9], the authors consider the incorrect indices in the previous support set and exclude them adaptively. From these researches, it can be seen that support priori can improve the recovery performance in massive MIMO, and compressive channel estimation can benefit from support priori by channel reciprocity in TDD system.

CS can also be used for channel estimation for FDD massive MIMO system in single-user (SU) scenario and multiple-user (MU) scenario. In single-user scenario, in [10], it makes use of the previous estimated support information and the burst structured sparsity for massive MIMO channel estimation. In [11], it separates the channel vector into a dense vector and a sparse vector and makes use of the previous channel to predict the dense vector by least squares algorithm and applies CS to estimate the sparse vector. In [12], it examines the impacts on the training overhead in FDD downlink channel estimation when previous channel support information is applied into a weighted $l_{1}$ minimization framework. In multiuser scenario, in [13], it proposes a close-loop pilot and CSIT feedback resource adaptation framework for MU massive MIMO, and the joint sparsity among users is used for compressive channel estimation. In [14], a two-stage weighted block $l_{1}$ minimization algorithm is proposed for downlink CSI estimation in FDD massive MIMO system, and the priori knowledge that MU channels share common supports is used. In [15], it takes a variational Bayesian inference-based approach for FDD channel estimation, and the partially joint sparsity shared by different users is captured. From the researches above, it can be seen that FDD massive MIMO channel estimation can benefit from the CS framework, and the support information from the previous estimated channel or from the structured sparsity among multiusers can improve the estimation performance.

On the other hand, in the FDD system, the propagation environment is almost the same for the uplink and downlink transmissions in short interval. The number of significant multipaths, path delays, and path angles are almost the same for uplink and downlink [16]. There exists reciprocity for uplink and downlink in massive MIMO in angular domain which can also be used for efficient compressive channel estimation. In [17], it explores the reciprocal channel characteristics of the dominant propagation path in FDD for channel feedback; however, the CS framework is not adopted. In [18], the joint overcomplete dictionary learning for uplink and downlink is proposed to get better channel estimation performance, and the dictionary learning is based on the assumption that the supports for uplink and downlink are common. It can be seen that reciprocity in angular domain exists, and the reciprocity can also be used in compressive channel estimation.

From the researches above, it can be concluded that the priori information, such as channel supports among neighboring antennas, or in adjacent time frames, or between uplink and downlink, can all improve the channel estimation performance. However, most of them rarely consider the basis mismatch effects which will deteriorate the sparsity. In [19], a sparsity-enhancing algorithm is discussed for compressive estimation of doubly selective multicarrier channels, while the leakage effect is discussed but not for massive MIMO. It is not practical to assume the common supports for uplink and downlink channels in the FDD system. On the other hand, the reciprocity for massive MIMO uplink and downlink channel in the angular domain is not discussed adequately.

In this paper, we present an efficient channel estimation algorithm in massive MIMO FDD system. Specially inspired by priori knowledge of channel reciprocity in angular domain for uplink and downlink channels, we propose a weighted SP channel estimation with the diagnosed supports from the uplink channel. Moreover, the impacts on the sparsity caused by basis mismatch and angle deviation in massive MIMO are discussed. According to [20], the radio paths are arriving in clusters with angle spread. By utilizing the cluster properties, we apply the DBSCAN algorithm (density-based spatial clustering of applications with noise) to extract the support information of the uplink channel, and a DBSCANbased support diagnosis algorithm is proposed to get the probable support of downlink channel, then a weighted subspace pursuit channel estimation is presented.

The contributions of the paper are listed as follows:

- A channel support diagnosis algorithm based on DBSCAN is proposed, in which the reciprocity between uplink and downlink channels in angular domain is used.

- A weighted SP algorithm is proposed for massive MIMO channel estimation, and the RIP-based performance analysis for weighted SP is proposed, which shows the superiority of the weighted SP compared with the standard SP.

The rest of the paper is organized as follows. The system model is described in Section 2. The support analysis of massive MIMO channel in angular or spatial domain is given out in Section 3. In Section 4, the weighted SP for massive MIMO channel estimation based on the diagnosed support is presented. The RIPbased performance analysis and simulations are illustrated in Sections 5 and 6. Finally, the conclusions are drawn in Section 7. 
Notation: $\boldsymbol{A}_{T}$ is a matrix composed of the columns of the matrix $A$ of the set $T . T^{c}$ symbolizes the complementary set of $T$. $\operatorname{resid}(\cdot)$ is the residue vector after projection operation, for example, $\operatorname{resid}(\mathbf{y}, \mathbf{A})=\mathbf{y}-$ $\boldsymbol{A} \boldsymbol{A}^{\dagger} \boldsymbol{y}$, where $\boldsymbol{A}^{\dagger}$ is the pseudoinverse of the matrix $\boldsymbol{A} . \delta_{k}$ is the parameter for $k$-RIP condition.

\section{System model}

We consider a scenario of a single user in massive MIMO FDD and assume the BS is equipped with $N$ antennas and the user terminal (UT) has a single antenna. For the uplink channel estimation, the uplink training received by the $\mathrm{BS}$ can be written as

$$
\boldsymbol{y}^{u}=\sqrt{\rho^{u}} \boldsymbol{h}^{u} \boldsymbol{a}+\boldsymbol{n}^{u}
$$

where $\boldsymbol{h}^{u} \in \mathbb{C}^{N \times 1}$ is the uplink channel, $\boldsymbol{a} \in \mathbb{C}^{1 \times T^{u}}$ is the uplink pilots, $T^{u}$ is the pilot length, $\rho^{u}$ is the uplink received power, $\boldsymbol{n}^{u} \in \mathbb{C}^{N \times T^{u}}$ is the received noise with each element to be i.i.d Gaussian with mean 0 and variance $\sigma^{2}$, and $y^{u} \in \mathbb{C}^{N \times T^{u}}$ is the received signal at BS.

For the downlink channel estimation in the FDD system, the BS transmits the pilots to UT. The UT receives the pilots and feeds the received signal back to the BS directly as in [21]. The received signal $y^{d}$ at the UT can be written as

$$
\boldsymbol{y}^{d}=\sqrt{\rho^{d}} \boldsymbol{A h}^{d}+\boldsymbol{n}^{d}
$$

where $\boldsymbol{h}^{d} \in \mathbb{C}^{N \times 1}$ is the downlink channel, $\boldsymbol{A} \in \mathbb{C}^{T^{d} \times N}$ is the downlink pilots, $T^{d}$ is the pilot length, $\rho^{d}$ is the downlink received power, $\boldsymbol{n}^{d} \in \mathbb{C}^{T^{d} \times 1}$ is the received noise with each element to be i.i.d Gaussian with mean 0 and variance $\sigma^{2}$, and $\boldsymbol{y}^{d} \in \mathbb{C}^{T^{d} \times 1}$ is the received signal at UT.

Since the massive MIMO channel exhibits spatial sparsity in angular/spatial domain, CS can be applied to the compressive channel estimation with much less measurements which means that $T^{d}<N$. In the compressive channel estimation, the uplink channel estimation in (1) can be represented as

$$
\hat{\boldsymbol{h}}_{a}^{u}=\operatorname{argmin}\left\|\boldsymbol{h}_{a}^{u}\right\|_{0}, \text { subject to }\left\|\boldsymbol{y}^{u}-\sqrt{\rho^{u}} \boldsymbol{D}^{u} \boldsymbol{h}_{a}^{u} \boldsymbol{a}\right\|_{2} \leq \varepsilon
$$

where $\boldsymbol{D}^{u} \in \mathbb{C}^{N \times N}$ is the channel basis matrix for uplink channel, $\boldsymbol{h}_{a}^{u}$ is the sparse spatial representation with $\boldsymbol{h}^{\boldsymbol{u}}=$ $\boldsymbol{D}^{u} \boldsymbol{h}_{a}^{u}$, and $\hat{\boldsymbol{h}}_{a}^{u}$ is the estimated uplink channel in the spatial domain. Similarly, the downlink channel estimation in (2) can be represented as

$$
\hat{\boldsymbol{h}}_{a}^{d}=\operatorname{argmin}\left\|\boldsymbol{h}_{a}^{d}\right\|_{0}, \text { subject to }\left\|\boldsymbol{y}^{d}-\sqrt{\rho^{d}} \boldsymbol{A} \boldsymbol{D}^{d} \boldsymbol{h}_{a}^{d}\right\|_{2} \leq \varepsilon
$$

where $D^{d} \in \mathbb{C}^{N \times N}$ is the channel basis matrix for downlink channel, $\boldsymbol{h}_{a}^{d}$ is the sparse representation with $\boldsymbol{h}^{d}=\boldsymbol{D}^{d} \boldsymbol{h}_{a}^{d}$, and $\hat{\boldsymbol{h}}_{a}^{d}$ is the estimated downlink channel in the spatial domain.

In most existing literature problems, (3) and (4) are solved separately. A more effective method is to solve (3) and (4) jointly. Channel support refers in particular to the set comprised by the locations of nonzero elements of channel representation in the angular/ spatial domain in this paper. In [18], it is assumed that $\boldsymbol{h}_{a}^{u}$ and $\boldsymbol{h}_{a}^{d}$ have the same supports and get the sparse solutions $\hat{\boldsymbol{h}}_{a}^{u}, \hat{\boldsymbol{h}}_{a}^{d}$ and the dictionary matrices $D^{u}, D^{d}$ iteratively. The uplink and downlink channel estimation benefits from the joint dictionary learning. However, the assumption of common supports of $\boldsymbol{h}_{a}^{u}$ and $\boldsymbol{h}_{a}^{d}$ is not practical because of basis mismatch and angle deviation between uplink and downlink. In the following, we will discuss the partial common supports of $\boldsymbol{h}_{a}^{u}$ and $\boldsymbol{h}_{a}^{d}$ based on the reciprocity in angular domain in massive MIMO channel and then propose a support diagnosis algorithm which can provide support priori information for compressive channel estimation.

\section{Channel supports in massive MIMO spatial channel}

We discuss the basis mismatch and leakage effects on the support in massive MIMO spatial channel first and then take the angle deviation between uplink and downlink into considerations for support analysis.

\subsection{Mismatch of MIMO channel basis}

In massive MIMO, the dominant physical paths between the transmitter and the receiver are relatively small compared to the number of antennas. For example, in the proposed channel model in the report 3GPP TS36.900 [12], the number of dominating physical path is six. So there is channel sparsity in angular domain. Similarly, as we did in [22], the massive MIMO channel can be given by

$$
\boldsymbol{H}=\sum_{i=1}^{p} a_{i} \boldsymbol{e}_{r}\left(\Omega_{r i}\right) \boldsymbol{e}_{t}\left(\Omega_{t i}\right)^{H}
$$

where $\boldsymbol{H}$ is the physical channel model of MIMO, $a_{i}$ is the attenuation of the $i$ th path, and $\theta_{t i}$ and $\theta_{r i}$ are the angle of departure and the angle of arrival $\left(\Omega_{t i}:=\cos \theta_{t i}\right.$, $\left.\Omega_{r i}:=\cos \theta_{r i}\right)$, respectively; $\boldsymbol{e}_{r}$ and $\boldsymbol{e}_{t}$ are the steering vectors of the receiving and transmitting antenna arrays, respectively, and are given by

$$
a_{i}:=\beta_{\mathrm{i}} \sqrt{N_{t} N_{r}} \exp \left(-\frac{j 2 \pi d_{i}}{\lambda_{c}}\right)
$$




$$
\begin{aligned}
& \mathbf{e}_{r}(\Omega):=\frac{1}{\sqrt{N_{r}}}\left[\begin{array}{llll}
1 & \exp \left(-j 2 \pi \Delta_{r} \Omega\right) & \cdots & \exp \left(-j 2 \pi\left(N_{r}-1\right) \Delta_{r} \Omega\right)
\end{array}\right]^{T} \\
& \mathbf{e}_{t}(\Omega):=\frac{1}{\sqrt{N_{t}}}\left[\begin{array}{llll}
1 & \exp \left(-j 2 \pi \Delta_{t} \Omega\right) & \cdots & \exp \left(-j 2 \pi\left(N_{t}-1\right) \Delta_{t} \Omega\right)
\end{array}\right]^{T}
\end{aligned}
$$

where $d_{i}$ and $\beta_{i}$ are the distance and large scale fading of the $i$ th path, respectively, and $\Delta_{r}$ and $\Delta_{t}$ are the normalized separation at the receiving and transmitting antenna arrays by the wavelength $\lambda_{c}$ of the transmitted signal, respectively.

We formulate the uplink and downlink channel basis matrices as in [22]:

$$
\begin{aligned}
\boldsymbol{U}_{t} & =\left[\boldsymbol{e}_{t}(0), \boldsymbol{e}_{t}\left(\frac{1}{L_{t}}\right), \cdots, \boldsymbol{e}_{t}\left(\frac{N_{t}-1}{L_{t}}\right)\right] \\
\boldsymbol{U}_{r} & =\left[\boldsymbol{e}_{r}(0), \boldsymbol{e}_{r}\left(\frac{1}{L_{r}}\right), \cdots, \boldsymbol{e}_{r}\left(\frac{N_{r}-1}{L_{r}}\right)\right]
\end{aligned}
$$

where $L_{t}=N_{t} \Delta_{t}$, and $L_{r}=N_{r} \Delta_{r}$. Then, the spatial massive MIMO channel can represented as

$$
\boldsymbol{H}^{\prime}=\boldsymbol{U}_{r}^{H} \boldsymbol{H} \boldsymbol{U}_{\boldsymbol{t}}
$$

where $\boldsymbol{H}^{\prime}$ is the MIMO channel in the angular/spatial domain. Then, the ( $k$ th, $l$ th) entry in channel matrix $\boldsymbol{H}^{\prime}$ is

$$
\begin{aligned}
h_{k l}^{\prime}= & \mathbf{e}_{\mathbf{r}}^{H}\left(\frac{k}{L_{r}}\right)\left(\sum_{i=1}^{P} a_{i} \mathbf{e}_{\mathbf{r}}\left(\Omega_{r i}\right) \mathbf{e}_{\mathbf{t}}\left(\Omega_{t i}\right)^{H}\right) \mathbf{e}_{\mathbf{t}}\left(\frac{l}{L_{t}}\right) \\
= & \sum_{i=1}^{P} a_{i} \mathbf{e}_{\mathbf{r}}^{H}\left(\frac{k}{L_{r}}\right) \mathbf{e}_{\mathbf{r}}\left(\Omega_{r i}\right) \mathbf{e}_{\mathbf{t}}\left(\Omega_{t i}\right)^{H} \mathbf{e}_{\mathbf{t}}\left(\frac{l}{L_{t}}\right) \\
& =\sum_{i=1}^{P} a_{i} f_{r}\left(\frac{k}{L_{r}}-\Omega_{r i}\right) \cdot f_{t}\left(\Omega_{t i}-\frac{l}{L_{t}}\right)
\end{aligned}
$$

where $f_{r}(\cdot)$ and $f_{t}(\cdot)$ have the forms as below:

$$
\begin{aligned}
& f_{r}\left(\Omega-\Omega^{\prime}\right)=\boldsymbol{e}_{r}^{H}(\Omega) \boldsymbol{e}_{r}\left(\Omega^{\prime}\right) \\
& f_{t}\left(\Omega-\Omega^{\prime}\right)=\boldsymbol{e}_{t}^{H}(\Omega) \boldsymbol{e}_{t}\left(\Omega^{\prime}\right)
\end{aligned}
$$

If $\Omega_{r j}$ and $\Omega_{t j}$ are not equal to $m / L_{r}$ and $n / L_{t}$ in (12), there are leakage effects on the entries of $\boldsymbol{H}^{\prime}$; then, we have

$$
h_{m n}^{\prime}=a_{j}+\sum_{i=1, i \neq j}^{P} a_{i} f_{r}\left(\frac{k}{L_{r}}-\Omega_{r i}\right) \cdot f_{t}\left(\Omega_{t i}-\frac{l}{L_{t}}\right)
$$

where the second part of (15) is the leakage effects of other paths. If the angle of departure (AOD) and angle of arrival (AOA) of the paths are integer multiples of $1 / L_{r}$ and $1 / L_{t}$, most of the entries of channel matrix in angular/ spatial domain are 0 . Otherwise, some entries of channel matrix in angular/spatial domain are not 0 because of the leakage effect even though there are no paths in the corresponding directions of the entries in channel matrix. It should be noticed that in the paper, the UT is equipped with single antenna; however, the analysis is also valid.

\subsection{Support difference between uplink and downlink massive MIMO channel}

In [23], it is assumed that the propagation environment is static during uplink-downlink transmissions in FDD; hence, the same multipaths, UE positions, and DOA for uplink are also valid for downlink; however, the assumption is not always valid in practical scenarios. As shown in Fig. 1, the DOA and AOA for uplink and downlink channels are not exactly the same but with some deviations. Based on the analysis of channel basis mismatch, the supports of uplink and downlink spatial channels are not exactly the same as shown in Fig. 1. However, the probable support of downlink channel can be inferred from the uplink channel, which we call support diagnosis in the paper.

In the practical scenario, the angle of transmission path for uplink and downlink will change slightly because of UE movement and environment change. On the other hand, in the spatial channel model in 3GPP TS36.900 report, it assumes that there are six paths, but it demonstrates that the mean angle spread is $5^{\circ}$ in suburban macrocell. Hence, it is practical to assume that the channel supports for uplink and downlink are partially common because of the angle deviation between uplink and downlink. Next, we will discuss the effects of angle deviation for uplink and downlink transmission on the channel supports.

Property 1: If the angle deviation for one path in uplink and downlink is $\Delta$ at BS as shown in Fig. 1, the deviation of the support center is bounded by $L_{r} \sqrt{2-2 \cos \Delta}$.

Proof: If we consider the angle difference for one transmission path and the AOA in the uplink at the BS is $\Omega_{t i}:=\cos \theta_{t i}$, the DOA in the downlink at the BS is $\Omega_{r i}$ : $=\cos \theta_{r i}$. In a practical scenario, $\theta_{t i}$ and $\theta_{r i}$ are not equal and with the difference $\Delta$. Then, we have

$$
\begin{aligned}
\cos \theta_{r i}-\cos \theta_{t i} & =\cos \left(\theta_{t i}+\Delta\right)-\cos \theta_{t i} \\
& =\sqrt{2-2 \cos \Delta} \sin \left(\phi-\theta_{t i}\right)
\end{aligned}
$$

where $\tan \phi=(\cos \Delta-1) / \sin \Delta$. Then, if the angle difference is $\Delta$, we have

$$
\left|\cos \theta_{r i}-\cos \theta_{t i}\right| \leq \sqrt{2-2 \cos \Delta}
$$

If the channel basis matrices are given as (9) and (10), the sampling interval in channel basis is $1 / L_{r}$; the 


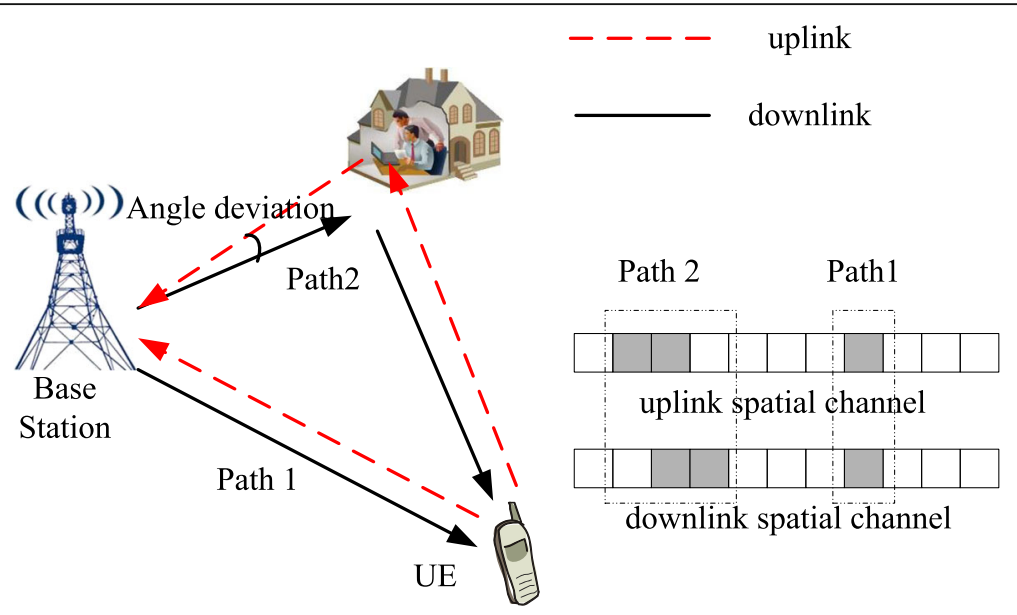

Fig. 1 Angle deviation and sparsity in spatial channel

position change of the support center will be $\left\lceil\mid \cos \theta_{r i}-\right.$ $\left.\cos \theta_{t i} \mid /\left(1 / L_{r}\right)\right\rceil$ and can be bounded as

$$
\left|\cos \theta_{r i}-\cos \theta_{t i}\right| /\left(1 / L_{r}\right) \leq L_{r} \sqrt{2-2 \cos \Delta}
$$

From property 1, it can be seen that support position is related to the antenna array size $L_{\mathrm{r}}$. The deviation of the support center is shown as Fig. 2b. In the following, we discuss the sparsity deterioration because of basis mismatch.

Property 2: The path angle arriving at the BS is $\theta$, and $\Omega=\cos \theta$. The channel basis matrices are defined as (9) and (10). If path angle $\Omega=i /_{L_{r}}, \mathrm{i} \in[1, \cdots, N]$, then in the spatial channel, only the $(i+1)$ th element is nonzero and there is not energy leakage; otherwise $\Omega=k / L_{r}$ $+\Omega^{\prime}, k \in[1, \cdots, N]$ and $\left|\Omega^{\prime}\right| \leq^{1} / 2 L_{r}$, then there is energy leakage to the neighborhood elements in the spatial channel, and at most a percentage of ${ }^{1} / \pi(\Delta k-1)$ of the energy is outside a region comprised by $[k-\Delta k, \cdots, k$, $\cdots, k+\Delta k]$ in the spatial channel.

Proof: For $\Omega=i / L_{r}$, there is no energy leakage which has been discussed in the previous section. Now, we consider $\Omega=k /_{L_{r}}+\Omega^{\prime}$ and analyze the energy leakage to the $\left(k^{\prime}+1\right)$ th atom in the channel basis. We define $\Delta_{\Omega}=\Omega-k^{\prime} / L_{r}=\Omega^{\prime}+k / L_{r}-k^{\prime} / L_{r}=\Omega^{\prime}+\Delta k / L_{r} \quad \Delta k=k-$ $k^{\prime}$, and we have

$$
\begin{aligned}
& f_{r}\left(\Delta_{\Omega}\right)=f_{r}\left(\Omega-k^{\prime} / L_{r}\right)=\boldsymbol{e}_{\boldsymbol{r}}(\Omega)^{H} \boldsymbol{e}_{\boldsymbol{r}}\left(k^{\prime} / L_{r}\right) \\
& =\frac{1}{N_{r}} \exp \left(j \pi \Delta_{r} \Delta_{\Omega}\left(N_{r}-1\right)\right) \frac{\sin \left(N_{r} \pi \Delta_{r} \Delta_{\Omega}\right)}{\sin \left(\pi \Delta_{r} \Delta_{\Omega}\right)}
\end{aligned}
$$

The absolute value of $f_{r}\left(\Delta_{\Omega}\right)$ is given by
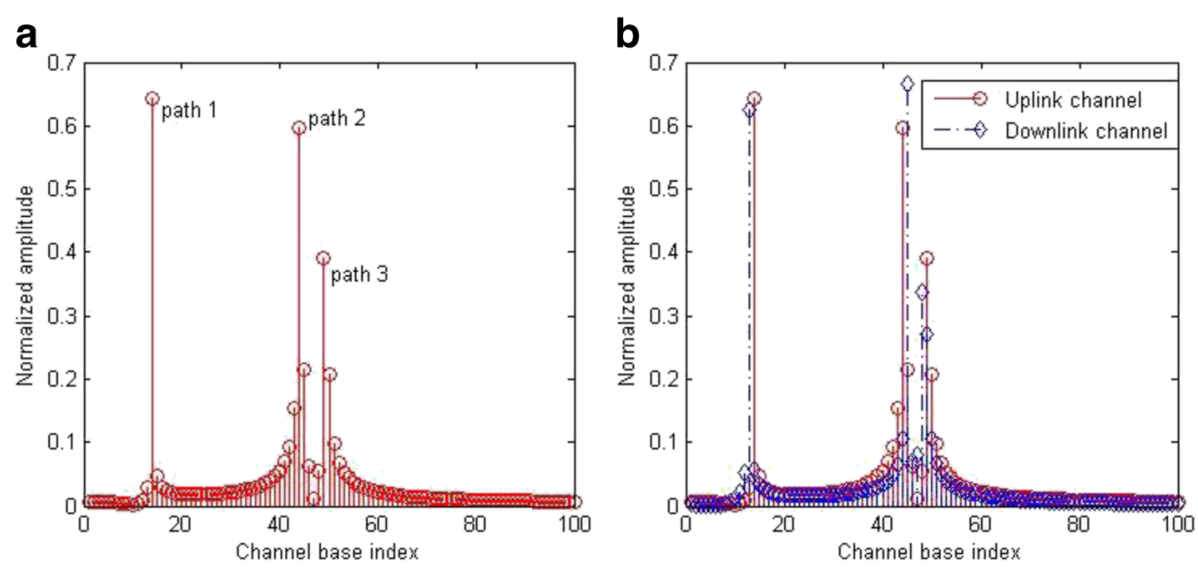

Fig. 2 a Sparsity in spatial channel and $\mathbf{b}$ uplink and downlink supports with angle deviation 


$$
\begin{aligned}
\left|f_{r}\left(\Delta_{\Omega}\right)\right| & =\left|f_{r}\left(\Omega^{\prime}+\Delta k^{\prime} / L_{r}\right)\right| \\
& =\frac{1}{N_{r}}\left|\frac{\sin \left(\pi L_{r}\left(\Omega^{\prime}+\Delta k^{\prime} / L_{r}\right)\right)}{\sin \left(\pi L_{r}\left(\Omega^{\prime}+\Delta k^{\prime} / L_{r}\right) / N_{r}\right)}\right|
\end{aligned}
$$

Let $\Gamma_{k^{\prime}+1}^{2}=\left|f_{r}\left(\Omega^{\prime}+\Delta k^{\prime} / L_{r}\right)\right|^{2}$, we have

$$
\Gamma_{k^{\prime}+1}^{2}=\frac{1}{N_{r}^{2}} \frac{\sin ^{2}\left(\pi L_{r}\left(\Omega^{\prime}+\Delta k^{\prime} / L_{r}\right)\right)}{\sin ^{2}\left(\pi L_{r}\left(\Omega^{\prime}+\Delta k^{\prime} / L_{r}\right) / N_{r}\right)}
$$

By utilizing the cyclic symmetry of $\Gamma_{k^{\prime}+1}^{2}$, the sum leakage energy which is outside the region comprised by $[k-\Delta k, \cdots, k, \cdots, k+\Delta k]$ in the spatial channel are given by

$$
\begin{gathered}
\Gamma_{\Delta k}^{2}+\ldots+\Gamma_{N_{r-\Delta k}}^{2}=\frac{2}{N_{r}^{2}} \sum_{i=\Delta k}^{N_{r} / 2} \frac{\sin ^{2}\left(\pi L_{r}\left(\Omega^{\prime}+i / L_{r}\right)\right)}{\sin ^{2}\left(\frac{\pi L_{r}\left(\Omega^{\prime}+i / L_{r}\right)}{N_{r}}\right)} \\
\leq \frac{2}{N_{r}^{2}} \sum_{i=k}^{N_{r} / 2} \frac{1}{\sin ^{2}\left(\frac{\pi L_{r}\left(\Omega^{\prime}+i / L_{r}\right)}{N_{r}}\right)} \\
\leq \frac{2}{N_{r}^{2}} \int_{\Delta k-1}^{N_{r} / 2} \frac{d i}{\sin ^{2}\left(\frac{\pi L_{r} i}{N_{r}}\right)} \\
=\frac{2}{\pi N_{r}}\left(\cot \left(\pi(\Delta k-1) / N_{r}\right)\right) \\
\leq \frac{1}{\pi(\Delta k-1)}
\end{gathered}
$$

Since $\sum_{i=1}^{N} \Gamma_{i}^{2}=1$ by the Parseval theory, property 2 is proofed.

From property 2, it can be seen that although there exists energy leakage because of basis mismatch, most of the energy is allocated around the support corresponding to the angle direction, and the leakage energy decreases in the rate of $\frac{1}{\pi(\Delta k-1)}$.

To illustrate these properties, we assume that the antenna number is 100; there are only three dominant paths. In Fig. 2, it can be seen that the channel basis index or socalled the channel taps in the spatial domain are located in cluster. If the channel basis matches the channel angle perfectly, the sparsity is relatively good such as path 1 in Fig. 2a; otherwise, the sparsity deteriorates such as path 2 and path 3. Meanwhile, if the angles of two paths are close, the amplitudes of the channel taps in spatial domain for them are superpositioned because of the leakage effects. In the ideal assumption, the AOA and DOA for uplink channel and downlink channel are common, but in the practical scenario, they are not the same. We assume the angle deviation for one path in uplink and downlink is within $5^{\circ}$ randomly as [20, 24]; then, as shown in Fig. 2b, it can be seen that the channel taps or channel supports for uplink and downlink in the spatial domain change slightly and are partially common as property 1 . In the following, we will utilize these properties for downlink spatial channel support diagnosis.

\section{Proposed methods}

In this section, the support diagnosis algorithm is first proposed based on the reciprocity in angular domain for uplink and downlink channels; then, the downlink massive MIMO channel estimation algorithm based on the diagnosed support is proposed.

\subsection{Channel support diagnosis algorithm}

In this subsection, we propose the channel support diagnosis algorithm based on the analysis of basis mismatch and support difference between the downlink channel and uplink channel. Although the AOA and DOA for uplink and downlink are not exactly the same, the multipath number is common for uplink and downlink. Inspired by the clustering property of channel support in spatial domain, for example, there are three clusters in Fig. 2b, we apply the DBSCAN (density-based spatial clustering of applications with noise) algorithm to extract the multipath information in the spatial domain. Then, we infer the probable channel supports of downlink channel. The details of the support diagnosis algorithm are presented in Algorithm 1.

Algorithm 1 Downlink spatial channel support diagnosis algorithm

Input: uplink spatial channel support set $\Omega^{u}=\operatorname{supp}\left(\widehat{\boldsymbol{h}}_{a}^{u}\right)$, the mean angle deviation $\theta$ for uplink and downlink with $\Delta=\cos \theta$, antenna number $N$ and antenna spacing $\Delta_{r}$ at BS with $L=N \Delta_{r}$.

Output: estimated downlink spatial channel support set $\Omega^{d}$

S1 (Coarse multipath number estimation): estimate the multipath number $n$ and the

cluster set for each path $\Omega_{i}^{u^{\prime}}, i \in[1,2, \cdots, n]$ by DBSCAN algorithm from uplink spatial

channel support set $\Omega^{u}$; the new clustered support set is $\Omega^{u^{\prime}}=\cup \Omega_{i}^{u^{\prime}}$.

S2 (Amending multipath number): sort the uplink spatial channel $\widehat{\boldsymbol{h}}_{a}^{u}$ in descending

order and select the first $n_{\max }$ elements; if the selected element is not included in $\Omega^{u^{\prime}}$,

then formulate a new clustering set $\Omega_{i^{\prime}}^{u^{\prime}}$ comprised by the excluded element. By processing all the $n_{\max }$ elements, and then get the amended multipath number $n^{\prime}$ and new clustering set $\Omega_{i}^{u^{i}}, i \in\left[1, \cdots, n^{\prime}\right]$.

S3 (Path central support estimation in spatial channel): each path is corresponding to one clustering set $\Omega_{i}^{u^{i}}$ in spatial channel, then the central support index in spatial channel for each path is estimated as $\lambda_{i}^{u}=\operatorname{floor}\left(\operatorname{average}\left(\Omega_{i}^{u^{\prime}}\right)\right)$, where $\operatorname{floor}(\cdot)$ is rounding operation and average $(\cdot)$ is averaging operation.

S4 (Downlink spatial channel support estimation): considering the angle deviation of each path for uplink and downlink, the deviation of central support in spatial channel for path $i$ is within $\left[\lambda_{i}^{u}-L \sqrt{2-2 \cos \Delta}, \cdots, \lambda_{i}^{u}+L \sqrt{2-2 \cos \Delta}\right]$. By considering the leakage effect, the support set for path $i$ is $\Omega_{i}^{d}=\left[\lambda_{i}^{u}-L \sqrt{2-2 \cos \Delta}-\Delta m, \cdots, \lambda_{i}^{u}+\right.$ $L \sqrt{2-2 \cos \Delta}+\Delta m]$, where $\Delta m$ is neighboring support number with leakage energy for the central support. Then the estimated downlink spatial channel support is $\Omega^{d}=\mathrm{U}$ $\Omega_{i}^{d}, i \in\left[1, \cdots, n^{\prime}\right]$.

In step S1, the estimated uplink spatial channel is utilized for path number estimation by DBSCAN 
algorithm. The DBSCAN algorithm is a data clustering algorithm proposed by Martin Ester which is widely used in machine learning [25]. It is a density-based clustering algorithm in which it groups together points that are closely allocated and marks those as outlier points which lie in low-density regions. In DBSCAN algorithm, the points are classified as core points, density reachable points, and outliers. A point $p$ is a core point if at least minPts points are within distance $\varepsilon$, and $\varepsilon$ is the maximum radius of the neighborhood from $p$. A point $q$ is reachable from $p$ if there is a path $p_{1}, \ldots, p_{n}$ with $p_{1}=p$ and $p_{n}=q$, where each $p_{i+1}$ is directly reachable from $p_{i}$. If $p$ is a core point, then it forms a cluster together with all points that are reachable from it. For example, in Fig. $4, p_{3}$ is reachable from $p_{1}$, and $p_{1}, p_{2}, p_{3}$, and $p_{4}$ are comprised as cluster 2 . The detailed algorithm can be found in [26].

By step S1, we can capture the multipaths in most conditions by DBSCAN except if the channel basis matches the path angle well or the leakage channel taps are not selected, for example, only one channel tap alone for the path is selected such as path 1 in Fig. 2a; then, by DBSCAN algorithm, this alone channel tap will be omitted because it is in the low-density region such as point $p_{n}$ in Fig. 3 which is the inherent characteristic of DBSCAN. In order to avoid this situation, we add these channel supports manually in step S2. In this way, we can capture most of the channel supports and get the multipath information.

In the spatial channel, each path is corresponding to one cluster set. If the channel basis matches the path angle well, then there is no energy leakage, and the corresponding cluster set has only one element; otherwise, there are multiple elements in the cluster set. In step S3, we estimate the central support for each path by averaging the index value of each cluster set. For example, in Fig. 2a, the central support is 44 for path 2 .

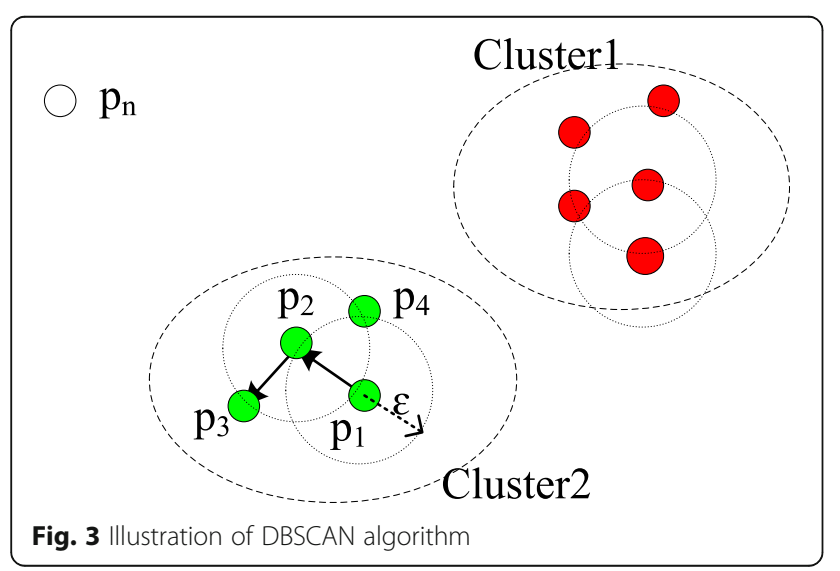

In step S4, we consider how to get the probable support of the downlink channel from the uplink channel support. If we consider the angle deviation between uplink and downlink, the change of channel tap index is within about $\pm L_{r} \sqrt{2-2 \cos \Delta}$ as property 1 . If the antenna number is 100 and antennas are half-wave allocated, then $L_{r}=50$. If the angle deviation is about $5^{\circ}$, the channel index deviation is about \pm 4.36 . From the leakage effect analysis in the previous section, at most a percentage of $1 / \pi(\Delta m-1)$ of the energy is located outside a rectangular neighborhood of the central channel support with interval of $\pm \Delta m$. For example, if we consider about $90 \%$ energy, then $\Delta m=4$.

We can diagnose the downlink channel supports in step S4 based on the uplink channel support information. The diagnosis procedure is as follows: firstly, we find the multipath number and the center support index $p_{i}$ of each path in the spatial domain; then, taking the angle deviations into account, it can be inferred that the center support index of each path change is within the range of $\left[p_{i}-L_{r} \sqrt{2-2 \cos \Delta}, p_{i}+L_{r} \sqrt{2-2 \cos \Delta}\right]$; following, taking the basis mismatch effect and $90 \%$ energy criterion into consideration, the probable support index is within the range of $\left[p_{i}-L_{r} \sqrt{2-2 \cos \Delta}-\Delta m, \cdots, p_{i}+L_{r}\right.$ $\sqrt{2-2 \cos \Delta}+\Delta m]$; then, we can get the probable downlink channel support set.

\subsection{Compressive downlink channel estimation with support priori}

Firstly, the BS utilizes CS recovery algorithm for the uplink channel estimation, and UT feeds back the received pilot signal to the $\mathrm{BS}$. At the $\mathrm{BS}$, the channel support diagnosis procedure is carried out as Algorithm 1. In order to integrate the support diagnosis information of downlink channel into the CS algorithm, we assign a weighting matrix $W=$ $\operatorname{diag}\left(\left[w_{1}, \cdots, w_{N}\right]\right)$ based on the diagnosed support information. The weights are given by

$$
w(i)=\left\{\begin{array}{l}
w_{1}=1, \text { if } i \in \Omega^{d} \\
w_{2}=\sigma, \text { if } i \notin \Omega^{d}
\end{array}\right.
$$

where $0<\sigma<1$ is the penalty parameter and $\Omega^{d}$ is the estimated downlink spatial channel support set. In the paper, we merge the weighting matrix into the subspace pursuit (SP) algorithm, and the details for the modified SP with support priori are presented in Algorithm 2. Compared with the standard SP, the main difference is the candidate support selection in step S1. In the standard SP, the $n$ largest magnitude entries are selected in the vector $\boldsymbol{A}^{H} \boldsymbol{V}_{k-1}$, while in the proposed weighted SP algorithm, the weighting matrix $W$ takes into consideration which contains the 
priori information from uplink channel. It should be noted that the diagnosed support information can be merged into other CS recovery algorithms equivalently.

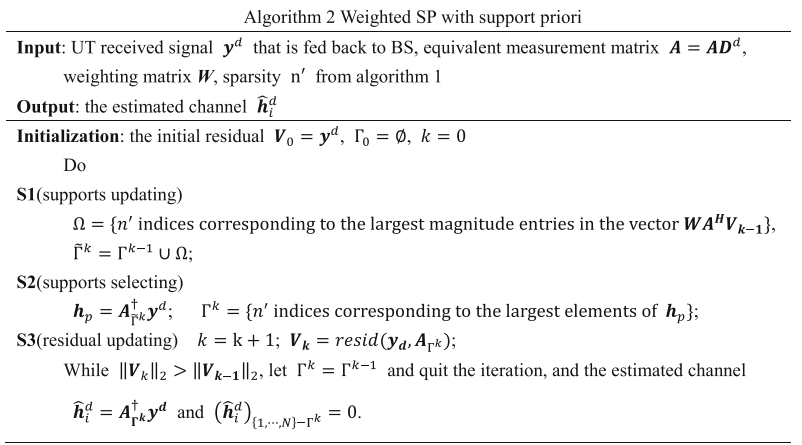

\section{RIP-based performance analyses}

In this section, we study the RIP-based performance of the proposed algorithm. The basic idea for the analysis mainly stems from Dai and Milenkovic in [27] but is with the consideration of the weights in (23). Compared with the standard SP algorithm, the main difference in the proposed algorithm is the support updating step (S1) in Algorithm 2. In the following, for brevity $y_{d}$ is denoted as $\mathbf{y}$ and $\boldsymbol{h}_{a}^{d}$ is denoted as $\boldsymbol{h}$.

The following analysis uses several propositions from [27]. We bring these in this subsection first to keep the analysis complete. According to the definition of residue vector of $\boldsymbol{V}_{k-1}$, we can get

$$
\begin{gathered}
\boldsymbol{V}_{k-1}=\operatorname{resid}\left(\boldsymbol{y}, \boldsymbol{A}_{\Gamma^{k-1}}\right) \\
=\operatorname{resid}\left(\boldsymbol{A}_{T-\Gamma^{k-1}} \boldsymbol{h}_{T-\Gamma^{k-1}}+\boldsymbol{A}_{T \cap \Gamma^{k-1}} \boldsymbol{h}_{T \cap \Gamma^{k-1}}+\boldsymbol{n}, \boldsymbol{A}_{\Gamma^{k-1}}\right) \\
=\operatorname{resid}\left(\boldsymbol{A}_{T-\Gamma^{k-1}} \boldsymbol{h}_{T-\Gamma^{k-1}}, \boldsymbol{A}_{\Gamma^{k-1}}\right)+\operatorname{resid}\left(\boldsymbol{A}_{T \cap \Gamma^{k-1}} \boldsymbol{h}_{T \cap \Gamma^{k-1}}, \boldsymbol{A}_{\Gamma^{k-1}}\right)+\operatorname{resid}\left(\boldsymbol{n}, \boldsymbol{A}_{\Gamma^{k-1}}\right) \\
=\operatorname{resid}\left(\boldsymbol{A}_{T-\Gamma^{k-1}} \boldsymbol{h}_{T-\Gamma^{k-1}}, \boldsymbol{A}_{\Gamma^{k-1}}\right)+\operatorname{resid}\left(\boldsymbol{n}, \boldsymbol{A}_{\Gamma^{k-1}}\right) \\
=\boldsymbol{A}_{T-\Gamma^{k-1}} \boldsymbol{h}_{T-\Gamma^{k-1}}+\boldsymbol{A}_{\Gamma^{k-1}} \boldsymbol{h}_{p, \Gamma^{k-1}}+\operatorname{resid}\left(\boldsymbol{n}, \boldsymbol{A}_{\Gamma^{k-1}}\right)
\end{gathered}
$$

where $T$ is the actual support of $\boldsymbol{h}, \boldsymbol{h}_{p, \Gamma^{k-1}}=-$ $\left(\boldsymbol{A}_{\Gamma^{k-1}}^{H} \boldsymbol{A}_{\Gamma^{k-1}}\right)^{-1} \boldsymbol{A}_{\Gamma^{k-1}}^{H} \boldsymbol{A}_{T-\Gamma^{k-1}} \boldsymbol{h}_{T-\Gamma^{k-1}}$. The fourth line in (24) is according to the definition of residual. The fifth line in (24) is according to the definition of projection. One can write

$$
\begin{aligned}
\boldsymbol{V}_{k-1} & =\boldsymbol{A}_{T \cup \Gamma^{k-1}} \boldsymbol{h}_{r, k-1}+\operatorname{resid}\left(\boldsymbol{n}, \boldsymbol{A}_{\Gamma^{k-1}}\right) \\
& =\left[\boldsymbol{A}_{T-\Gamma^{k-1}}, \boldsymbol{A}_{\Gamma^{k-1}}\right]\left[\begin{array}{c}
\boldsymbol{h}_{T-\Gamma^{k-1}} \\
\boldsymbol{h}_{p, \Gamma^{k-1}}
\end{array}\right]+\operatorname{resid}\left(\boldsymbol{n}, \boldsymbol{A}_{\Gamma^{k-1}}\right) .
\end{aligned}
$$

Proposition 1 [(25) in [27]]: $\left\|\boldsymbol{h}_{p, \Gamma^{k-1}}\right\|_{2} \leq \frac{\delta_{2 k}}{1-\delta_{2 k}}\left\|\boldsymbol{h}_{T-\Gamma^{k-1}}\right\|_{2}$, where $T$ is the correct support set and $\Gamma^{k-1}$ is the estimated support during the $(k-1)$ th iteration in step (S3) of Algorithm 2.

Proposition 2 [(16) in [27]]: $\left\|\boldsymbol{h}_{T-\Gamma^{k}}\right\|_{2} \leq \frac{1+\delta_{3 k}}{1-\delta_{3 k}}\left\|\boldsymbol{h}_{T-\tilde{\Gamma}^{k}}\right\|_{2}$ $+\frac{2}{1-\delta_{3 k}}\|\boldsymbol{n}\|_{2}$, where $T$ is the actual support set and $\tilde{\Gamma}^{k}$ and $\Gamma^{k}$ are the support sets during the $k$ th iteration in steps S1 and S3 of Algorithm 2, respectively.

In step S4 of Algorithm 2, the residue vector is calculated after the new support set is obtained in step S2. The residue vector is shown as (24). Proposition 1 and 2 give out the inequalities that $\boldsymbol{h}_{T-\Gamma^{k-1}}$ and $\boldsymbol{h}_{p, \Gamma^{k-1}}$ in (24) will satisfy. Since in our proposed algorithm only step S1 is different from the standard SP, propositions 1 and 2 can be applied directly. Then, we will consider the impacts of step S1 only on the estimation error.

Theorem 3: By using the weighing matrix $W$ in (23), the proposed SP solution at the $l$ th iteration satisfies the inequality as

$$
\begin{aligned}
& \left\|\boldsymbol{h}_{T-\Gamma^{k}}\right\|_{2} \leq \sqrt{\frac{w_{1}^{2}+w_{2}^{2}}{w_{1}^{2}}} \frac{\delta_{3 k}\left(1+\delta_{3 k}\right)}{\left(1-\delta_{3 k}\right)^{3}}\left\|\boldsymbol{h}_{T-\Gamma^{k-1}}\right\|_{2} \\
& +\frac{4\left(1+\delta_{3 k}\right)}{\left(1-\delta_{3 k}\right)^{2}}\|\boldsymbol{n}\|_{2} .
\end{aligned}
$$

Proof: According to the definition of $\Omega$ in $\mathrm{S} 1$ in Algorithm 2, then

$$
\left\|\boldsymbol{W}_{\Omega} \boldsymbol{A}_{\Omega}^{H} \boldsymbol{V}_{k-1}\right\|_{2} \geq\left\|\boldsymbol{W}_{\mathrm{T}} \boldsymbol{A}_{T}^{H} \boldsymbol{V}_{k-1}\right\|_{2}
$$

Removing the common columns between $\Omega$ and $\mathrm{T}$ on both sides of (25) and applying $\left\|\operatorname{resid}\left(\boldsymbol{n}, \boldsymbol{A}_{\Gamma^{k-1}}\right)\right\|_{2} \leq\|\boldsymbol{n}\|_{2}$ from the definition of residue operation, we arrive at

$$
\begin{gathered}
\left\|\boldsymbol{W}_{\Omega-\mathrm{T}} \boldsymbol{A}_{\Omega-\mathrm{T}}^{H} \boldsymbol{V}_{k-1}\right\|_{2} \geq\left\|\boldsymbol{W}_{\mathrm{T}-\Omega} \boldsymbol{A}_{T-\Omega}^{H} \boldsymbol{V}_{k-1}\right\|_{2} \\
\geq\left\|\boldsymbol{W}_{\mathrm{T}-\Omega} \boldsymbol{A}_{\mathrm{T}-\Omega}^{H} \boldsymbol{A}_{T \cup \Gamma^{k-1}} \boldsymbol{h}_{r, k-1}\right\|_{2}\left\|\boldsymbol{W}_{\mathrm{T}-\Omega} \boldsymbol{A}_{\mathrm{T}-\Omega}^{H} \operatorname{resid}\left(\boldsymbol{n}, \boldsymbol{A}_{\Gamma^{k-1}}\right)\right\|_{2} \\
\geq\left\|\boldsymbol{W}_{\mathrm{T}-\Omega} \boldsymbol{A}_{\mathrm{T}-\Omega}^{H} \boldsymbol{A}_{T \cup \Gamma^{k-1}} \boldsymbol{h}_{r, k-1}\right\|_{2}-w_{1} \sqrt{1+\delta_{k}}\left\|\operatorname{resid}\left(\boldsymbol{n}, \boldsymbol{A}_{\Gamma^{k-1}}\right)\right\|_{2} \\
\geq\left\|\boldsymbol{W}_{\mathrm{T}-\Omega} \boldsymbol{A}_{\mathrm{T}-\Omega}^{H} \boldsymbol{A}_{T \cup \mathrm{I}^{k-1}} \boldsymbol{h}_{r, k-1}\right\|_{2}-w_{1} \sqrt{1+\delta_{k}}\|\boldsymbol{n}\|_{2}
\end{gathered}
$$

The second line of (26) is from the triangle inequality and the expression of $V_{k-1}$. The third line is from the definition of $W$ in (23) and RIP property. On the other hand, by applying the triangle inequality, we have

$$
\begin{gathered}
\left\|\boldsymbol{W}_{\Omega-\mathrm{T}} \boldsymbol{A}_{\Omega-\mathrm{T}}^{H} \boldsymbol{V}_{k-1}\right\|_{2} \leq\left\|\boldsymbol{W}_{\Omega-\mathrm{T}} \boldsymbol{A}_{\Omega-\mathrm{T}}^{H} \boldsymbol{A}_{T \cup \mathrm{r}^{k-1}} \boldsymbol{h}_{r, k-1}\right\|_{2}+\left\|\boldsymbol{W}_{\Omega-\mathrm{T}} \boldsymbol{A}_{\Omega-\mathrm{T}}^{H} \operatorname{resid}\left(\boldsymbol{n}, \boldsymbol{A}_{\mathrm{\Gamma}^{k-1}}\right)\right\|_{2} \\
\leq\left\|\boldsymbol{W}_{\Omega-\mathrm{T}} \boldsymbol{A}_{\Omega-\mathrm{T}}^{H} \boldsymbol{A}_{T \cup \mathrm{T}^{k-1}} \boldsymbol{h}_{r, k-1}\right\|_{2}+w_{1} \sqrt{1+\delta_{k}\|\boldsymbol{n}\|_{2}}
\end{gathered}
$$

Combining (26) and (27), we have

$$
\begin{aligned}
& \left\|\boldsymbol{W}_{\Omega-\mathrm{T}} \boldsymbol{A}_{\Omega-\mathrm{T}}^{H} \boldsymbol{A}_{T \cup \mathrm{\Gamma}^{k-1}} \boldsymbol{h}_{r, k-1}\right\|_{2} \\
& \quad+2 w_{1} \sqrt{1+\delta_{k}}\|\boldsymbol{n}\|_{2} \geq\left\|\boldsymbol{W}_{\mathrm{T}-\Omega} \boldsymbol{A}_{\mathrm{T}-\Omega}^{H} \boldsymbol{A}_{T \cup \mathrm{r}^{k-1}} \boldsymbol{h}_{r, k-1}\right\|_{2}
\end{aligned}
$$

Then, we have 


$$
\begin{aligned}
& \left\|\boldsymbol{W}_{\Omega-\mathrm{T}} \boldsymbol{A}_{\Omega-\mathrm{T}}^{H} \boldsymbol{A}_{T \cup \mathrm{\Gamma}^{k-1}-\boldsymbol{h}_{r, k-1}}\right\|_{2}^{2}=\left\|\boldsymbol{W}_{(\Omega-\mathrm{T}) \cap \hat{T}} \boldsymbol{A}_{(\Omega-\mathrm{T}) \cap \hat{T}}^{H} \boldsymbol{A}_{T \cup \mathrm{r}^{k-1}} \boldsymbol{h}_{r, k-1}\right\|_{2}^{2} \\
& +\left\|\boldsymbol{W}_{(\Omega-\mathrm{T})-\hat{T}} \boldsymbol{A}_{(\Omega-\mathrm{T})-\hat{T}}^{H} \boldsymbol{A}_{T \cup \mathrm{r}^{k-1}} \boldsymbol{h}_{r, k-1}\right\|_{2}^{2}=w_{1}^{2}\left\|\boldsymbol{A}_{(\Omega-\mathrm{T}) \cap \hat{T}}^{H} \boldsymbol{A}_{T \cup \mathrm{r}^{k-1}} \boldsymbol{h}_{r, k-1}\right\|_{2}^{2} \\
& +w_{2}^{2}\left\|\boldsymbol{A}_{(\Omega-\mathrm{T})-\hat{T}}^{H} \boldsymbol{A}_{T \cup \mathrm{r}^{k-1} \boldsymbol{h}_{r, k-1}}\right\|_{2}^{2} \leq w_{1}^{2} \delta_{3 k}^{2}\left\|\boldsymbol{h}_{r, k-1}\right\|_{2}^{2}+w_{2}^{2} \delta_{3 k}^{2}\left\|\boldsymbol{h}_{r, k-1}\right\|_{2}^{2} \\
& =\left(w_{1}^{2}+w_{2}^{2}\right) \delta_{3 k}^{2}\left\|\boldsymbol{h}_{r, k-1}\right\|_{2}^{2}
\end{aligned}
$$

where $\hat{T}$ is the diagnosed support set and the weight is given by (23). Similarly, we have

$$
\begin{aligned}
& \left\|\boldsymbol{W}_{\mathrm{T}-\Omega} \boldsymbol{A}_{\mathrm{T}-\Omega}^{H} \boldsymbol{A}_{T \mathrm{U}^{k-1}-\boldsymbol{h}_{r, k-1}}\right\|_{2}^{2} \geq\left\|\boldsymbol{W}_{\mathrm{T}-\left(\Omega+\mathrm{r}^{k-1}\right)} \boldsymbol{A}_{\mathrm{T}-\left(\Omega+\mathrm{r}^{\mathrm{k}-1}\right)}^{H} \boldsymbol{A}_{T \mathrm{r}^{k-1}-\boldsymbol{h}_{r, k-1}}\right\|_{2}^{2}
\end{aligned}
$$

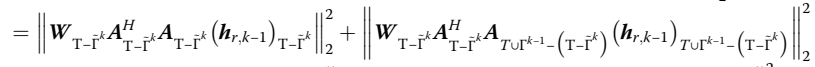

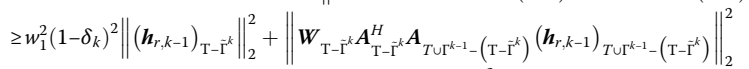

$$
\begin{aligned}
& \geq w_{1}^{2}\left(1-\delta_{k}\right)^{2}\left\|\left(\boldsymbol{h}_{r, k-1}\right)_{\mathrm{T}-\mathrm{I}^{k}}\right\|_{2}^{2}
\end{aligned}
$$

where the second line in (30) is according to step S1 in Algorithm 2 and the third line is according to Algorithm 1 and RIP properties.

Combining (29) and (30) into (28), we can get

$$
\begin{aligned}
& \sqrt{w_{1}^{2}+w_{2}^{2}} \delta_{3 k}\left\|\boldsymbol{h}_{r, k-1}\right\|_{2} \\
& \quad+2 w_{1} \sqrt{1+\delta_{k}}\|\boldsymbol{n}\|_{2} \geq w_{1}\left(1-\delta_{k}\right)\left\|\left(\boldsymbol{h}_{r, k-1}\right)_{\mathrm{T}-(\Omega+\Gamma)}\right\|_{2}
\end{aligned}
$$

Noting the explicit form of $\left\|\boldsymbol{h}_{k-1}\right\|_{2}$ and applying the triangle inequality, one has

$$
\begin{gathered}
\left\|\boldsymbol{h}_{r, k-1}\right\|_{2} \leq\left\|\boldsymbol{h}_{T-\Gamma^{k-1}}\right\|_{2}+\left\|\boldsymbol{h}_{p, \Gamma^{k-1}}\right\|_{2} \\
\leq\left(1+\frac{\delta_{2 k}}{1-\delta_{2 k}}\right)\left\|\boldsymbol{h}_{T-\Gamma^{k-1}}\right\|_{2} \\
\leq \frac{1}{1-\delta_{3 k}}\left\|\boldsymbol{h}_{T-\Gamma^{k-1}}\right\|_{2}
\end{gathered}
$$

where the second line in (32) is obtained by using proposition 1. Note that $\boldsymbol{V}_{k-1}=\boldsymbol{A}_{T \cup \Gamma^{k-1}} \boldsymbol{h}_{r, k-1}+$ resid $\left(\boldsymbol{n}, \boldsymbol{A}_{\Gamma^{k-1}}\right)=\left[\boldsymbol{A}_{T-\Gamma^{k-1}} \boldsymbol{A}_{\Gamma^{k-1}} \cdot\left[\begin{array}{c}\boldsymbol{h}_{T-\Gamma^{k-1}} \\ \boldsymbol{h}_{p, \Gamma^{k-1}}\end{array}\right]+\operatorname{resid}\left(\boldsymbol{n}, \boldsymbol{A}_{\Gamma^{k-1}}\right)\right.$, then $\left(\boldsymbol{h}_{r, k-1}\right)_{\mathrm{T}-(\Omega+\Gamma)}=\boldsymbol{h}_{T-\Gamma^{k}}$

Then, we get

$$
\left\|\boldsymbol{h}_{T-\mathrm{T}^{k-1}}\right\|_{2} \leq \sqrt{\frac{w_{1}^{2}+w_{2}^{2}}{w_{1}^{2}}} \frac{\delta_{3 k}}{\left(1-\delta_{3 k}\right)^{2}}\left\|\boldsymbol{h}_{T-\mathrm{r}^{k-1}}\right\|_{2}+\frac{2 \sqrt{1+\delta_{k}}}{1-\delta_{k}}\|\boldsymbol{n}\|_{2}
$$

Combining proposition 2 with (33), we complete the proof.

In the standard SP algorithm as shown in [27], at the $l$ th iteration it satisfies

$$
\left\|\boldsymbol{h}_{T-\Gamma^{k}}\right\|_{2} \leq \frac{2 \delta_{3 k}\left(1+\delta_{3 k}\right)}{\left(1-\delta_{3 k}\right)^{3}}\left\|\boldsymbol{h}_{T-\Gamma^{k-1}}\right\|_{2}+\frac{4\left(1+\delta_{3 k}\right)}{\left(1-\delta_{3 k}\right)^{2}}\|\boldsymbol{n}\|_{2}
$$

As in the weighting matrix (23), $\sqrt{\frac{w_{1}^{2}+w_{2}^{2}}{w_{1}^{2}}}<\sqrt{2}$, so the proposed algorithm can converge faster than the standard SP and have better estimation accuracy. When $\delta_{3 k}=$ 0.083 as required in [27] and assuming $w_{1}=w_{2}=1$ for the worst case, in our proposed algorithm, we have $\left\|\boldsymbol{h}_{T-\Gamma^{k}}\right\|_{2} \leq 0.164\left\|\boldsymbol{h}_{T-\Gamma^{k-1}}\right\|_{2}+5.152\|n\|_{2}$ while in the standard SP $\left\|\boldsymbol{h}_{T-\mathrm{T}^{k}}\right\|_{2} \leq 0.232\left\|\boldsymbol{h}_{T-\mathrm{K}^{k-1}}\right\|_{2}+5.152\|n\|_{2}$. When $\sigma=0.1$ in the proposed algorithm, we have $\left\|\boldsymbol{h}_{T-\Gamma^{k}}\right\|_{2} \leq 0.117\left\|\boldsymbol{h}_{T-\Gamma^{k-1}}\right\|_{2}+5.152\|n\|_{2}$. In this way, our proposed algorithm converges faster than the standard SP and has better recovery performance. In other words, the restriction of $\delta_{3 k}$ is weakened in the proposed algorithm. The performance improvement can be explained by that the SP algorithm can benefit from the priori support information.

\section{Simulation results}

In this section, simulations are carried out to evaluate the performance of the proposed downlink channel estimations with priori information. The BS is equipped with 100 antennas, and the UT is equipped with a single antenna. The channel model is generated according to spatial MIMO channel in 3GPP TS36.900. The same SNR is assumed for both uplink and downlink. Since we focus on the impacts of the priori information on the performance of downlink channel estimation, the gain of multiple pilots in uplink channel is not discussed, and the pilot length is set to 1 . The estimation accuracy of uplink channel can benefit from the increase of pilot number.

In Fig. 4, the penalty value $\sigma$ in the weighting matrix is 0.1 , the downlink pilot length is 50 , and the SNR for uplink and downlink are equal. We compare the performance of downlink channel estimation among four algorithms ((1)SP algorithms with no priori information as done in $[4,14]$, (2) weighted SP with the same supports as uplink as in [18], (3) weighted SP with the proposed diagnosed supports, and (4) standard iteratively reweighted least squares (IRLS) in [28]). It can be seen that in the low-SNR region algorithms, (1), (2), and (3) have almost the same MSE performance; the performance of algorithm (4) fluctuates around them. However, in the high-SNR region, the proposed algorithm has better performance, which can be explained by that the support information from uplink channel is more accurate in the high-SNR region since we assume the common SNR for uplink and downlink. The proposed support diagnosis information can improve the channel estimation MSE because it considers the basis mismatch, 


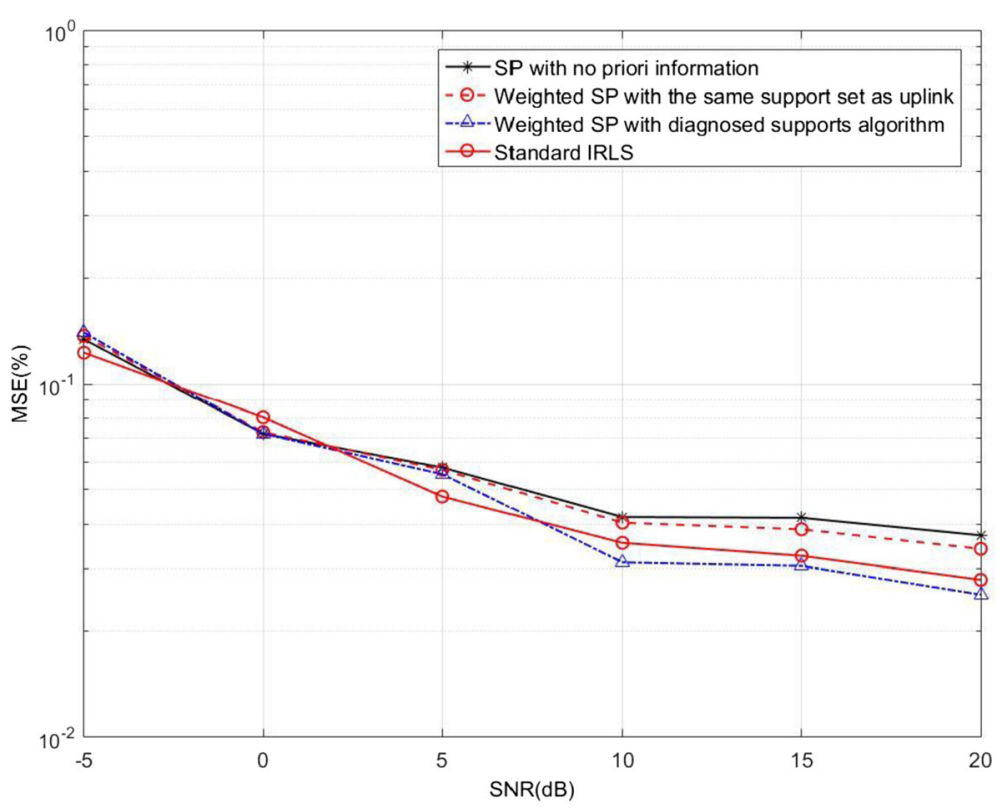

Fig. 4 MSE performance of the weighted SP recovery based on the diagnosed supports

the leakage effect, and the slight deviation of AOA and DOA for uplink and downlink.

In Fig. 5, the penalty value $\sigma$ in the weighting matrix is 0.1. We compare the performance of the downlink channel estimation with different pilot lengths. It can be seen that with the increase of the downlink pilot length, the MSE performance for the four algorithms ((1) SP algorithms with no priori information as done in $[4,14],(2)$ weighted SP with the same supports as uplink in [18],
(3) weighted SP with the proposed diagnosed supports, and (4) standard iteratively reweighted least squares (IRLS) in [28]) are all improved which are in accordance with the results in the CS theory. The proposed recovery algorithm with diagnosed support has the best recovery performance and has higher MSE improvement gain by pilot increasing in the high-SNR region.

In Fig. 6, we compare the performance of downlink channel estimation with different penalty values in

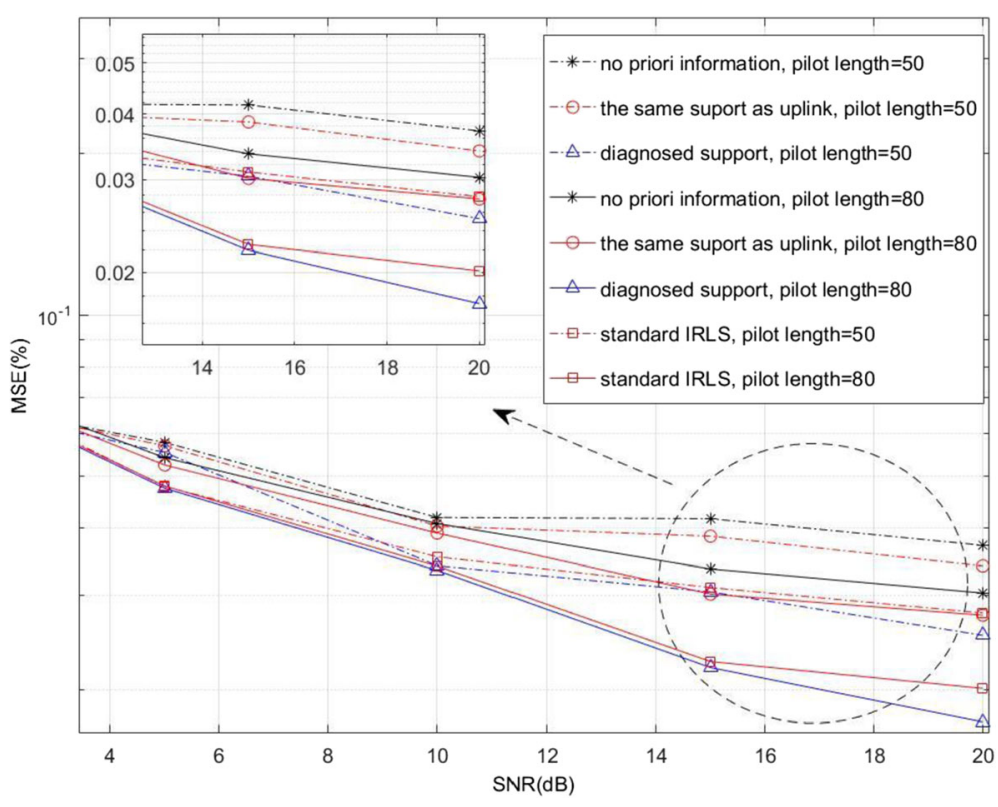

Fig. 5 Weighted SP recovery based on the diagnosed supports with different pilot lengths 


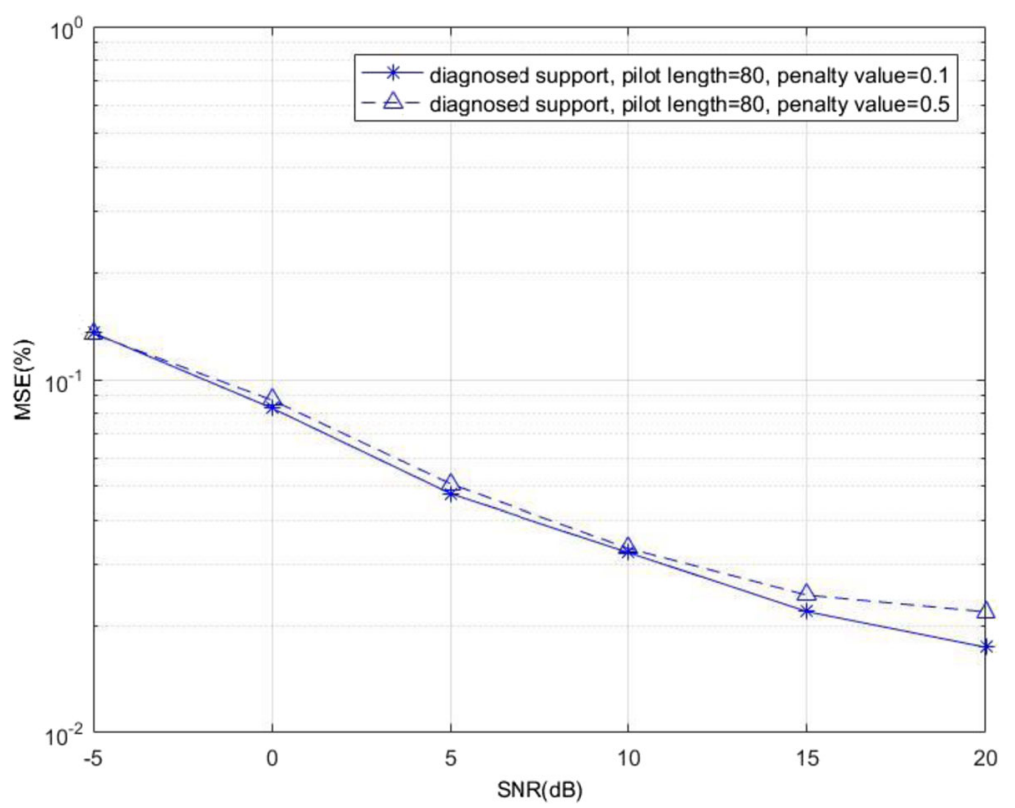

Fig. 6 Weighted SP recovery based on the diagnosed supports with different penalty values

weighting matrix in the proposed support diagnosis algorithm. It can be seen that weighting matrix with smaller penalty value in the proposed algorithm has better performance especially in the high-SNR region.

From the simulation results, above it can be concluded that (1) the uplink channel can offer support information for downlink channel estimation. The support information is useful for the downlink channel recovery. (2) The assumption of common support for downlink and uplink channels is not practical. The estimation accuracy will deteriorate if the support difference is ignored. The proposed support diagnosis algorithm considers the basis mismatch and angle deviation for uplink and downlink which can improve the downlink channel estimation further. (3) The weighting matrix value is important for the proposed weighted SP algorithm; smaller penalty value in weighting matrix is preferred especially in the highSNR region. In brief, the proposed support diagnosis algorithm is sufficient and beneficial for the downlink compressive channel estimation.

\section{Conclusions}

In this paper, we propose a downlink compressive channel estimation based on weighted SP for FDD massive MIMO systems, and the weighted SP makes use of the priori support information to improve the estimation performance. The reciprocity between uplink and downlink channels in angular domain is used for diagnosing the support priori information of downlink channel. The proposed support diagnosis algorithm considers the basis mismatch, leakage effect, and angle deviation for uplink and downlink channels and applies the DBSCAN algorithm used in machine learning to the channel support diagnosis. RIP-based analysis shows a better convergence and error performance of the proposed algorithm compared with the standard SP. Simulation results verify that the proposed algorithm improves the downlink channel estimation accuracy compared to the IRLS, the SP algorithm which does not utilize the uplink priori information, and the weighted SP which assumes that the downlink and uplink channels have common supports.

\section{Abbreviations}

AOA: Angle of arrival; AOD: Angle of departure; BS: Base station; CS: Compressed sensing; CSI: Channel state information; DBSCAN: Densitybased spatial clustering of applications with noise; FDD: Frequency division duplexing; IRLS: Iteratively reweighted least squares; LTE: Long-term evolution; MBP: Modified basis pursuit; MIMO: Multiple-input multiple-output; MU: Multiple user; OMP: Orthogonal matching pursuit; RIP: Restricted isometry property; SP: Subspace pursuit; SU: Single user; TDD: Time division duplexing; UT: User terminal

\section{Acknowledgements}

This work is supported in part by the National Science Foundation of China (No.61601509) and the China Postdoctoral Science Foundation Grant

(No.2016M603045). Thanks for the reviewers' comments.

\section{Funding}

The National Science Foundation of China (Grant No.61601509) and the China Postdoctoral Science Foundation (Grant No.2016M603045) are supporting the simulations and data analyses.

\section{Authors' contributions}

All authors discussed the experiments; WL performed the experiments and wrote the paper. YW, QF and SP have made some useful comments on the paper. All authors have read and approved the final manuscript. 


\section{Authors' information}

Wei Lu received a Ph.D degree in communications and information system from Huazhong University of Science and Technology, China, in 2013. Now, he is a lecturer in the Air Force Early Warning Academy in China. His current research interests focus on compressed sensing and signal processing. Yongliang Wang received a Ph.D degree in signal processing from Xidian University, China, in 1994. Now, he is a professor in the Air Force Early Warning Academy in China. His current research interests focus on STAP. Qiqing Fang is an associate professor in the Air Force Early Warning Academy in China. His current research interests focus on operations research and management. Shixin Peng received a Ph.D degree in communications and information system from Huazhong University of Science and Technology, China, in 2015. Now, he is a postdoctor in Central China Normal University. His current research interests focus on wireless communications and signal processing.

\section{Competing interests}

The authors declare that they have no competing interests.

\section{Publisher's Note}

Springer Nature remains neutral with regard to jurisdictional claims in published maps and institutional affiliations.

\section{Author details \\ 'Air Force Early Warning Academy, Wuhan, China. ${ }^{2}$ National Engineering Research Centre for E-Learning, Central China Normal University, Wuhan, China.}

Received: 18 December 2017 Accepted: 26 April 2018

Published online: 10 May 2018

\section{References}

1. X Liu, F Li, ZY Na, Optimal resource allocation in simultaneous cooperative spectrum sensing and energy harvesting for multichannel cognitive radio. IEEE Access 5, 3801-3812 (2017)

2. G Wunder, $\mathrm{H}$ Boche, $T$ Strohme, $P$ Jung, Sparse signal processing concepts for efficient 5 G system design. IEEE Access 3, 195-208 (2015)

3. L Lu, GY Li, AL Swindlehurst, A Ashikhmin, R Zhang, An overview of massive MIMO: benefits and challenges. IEEE J. Sel. Topics Signal Process. 8(5), 742758 (2014)

4. X Rao, VKN Lau, Distributed compressive CSIT estimation and feedback for FDD multi-user massive MIMO systems. IEEE Trans. Signal Process. 62(12), 3261-3271 (2014)

5. $\quad$ N Vaswani, W Lu, Modified-CS: modifying compressive sensing for problems with partially known support. IEEE Trans. Signal Process. 58(9), 4595-4607 (2010)

6. M Masood, LH Afify, TY Al-Naffouri, Efficient coordinated recovery of sparse channels in massive MIMO. IEEE Trans. Signal Process. 63(1), 104-118 (2015)

7. $\quad Y$ Nan, L Zhang, X Sun, Weighted compressive sensing based uplink channel estimation for time division duplex massive multi-input multioutput systems. IET Commun. 11(3), 355-361 (2017)

8. Y Nan, L Zhang, X Sun, Efficient downlink channel estimation scheme based on block-structured compressive sensing for TDD massive MU-MIMO systems. IEEE Wireless Commun. Lett. 4(4), 345-348 (2015)

9. X Rao, V Lau, Compressive sensing with priori support quality information and application to massive MIMO channel estimation with temporal correlation. IEEE Trans. Signal Process. 63(18), 4914-4924 (2015)

10. A Liu, VKN Lau, W Dai, Exploiting burst-sparsity in massive MIMO with partial channel support information. IEEE Trans. Wirel. Commun. 15(11), 7820-7830 (2016)

11. YH Han, JW Lee, DJ Love, Compressed sensing-aided downlink channel training for FDD massive MIMO systems. IEEE Trans. Commun. 65(7), 28522862 (2017)

12. JC Shen, J Zhang, E Alsusa, KB Letaief, Compressed CSI acquisition in FDD massive MIMO: how much training is needed? IEEE Trans. Wirel. Commun. 15(6), 4145-4156 (2016)

13. A Liu, F Zhu, VKN Lau, Closed-loop autonomous pilot and compressive CSIT feedback resource adaptation in multi-user FDD massive MIMO systems. IEEE Trans. Signal Process. 65(1), 173-183 (2017)

14. CC Tseng, J Y Wu, T S Lee, 2016 IEEE $27^{\text {th }}$ Annual IEEE International Symposium on Personal, Indoor and Mobile Radio Communications.
Compressive downlink CSI estimation for FDD massive MIMO systems: a weighted block L1-minimization approach (2016)

15. X Cheng, J Sun, S Li, Channel estimation for FDD multi-user massive MIMO: a variational Bayesian inference-based approach. IEEE Trans. Wirel. Commun. 16(11), 7590-7602 (2017)

16. K Hugl, K Kalliola, J Laurila, in Proc. COST 273 Technical Document TD. Spatial reciprocity of uplink and downlink radio channel in FDD systems, vol 66 (2002), p. 7

17. U Ugurlu, R Wichman, CB Ribeiro, C Wijting, A multipath extraction-based CSI acquisition method for FDD cellular networks with massive antenna arrays. IEEE Trans. Wirel. Commun. 15(4), 2940-2953 (2016)

18. Y Ding, B D Rao, in IEEE Global Conference on Signal and Information Processing, Channel estimation using joint dictionary learning in FDD massive MIMO system(2015), 185-189

19. G TaubÖck, F Hlawatsch, D Eiwen, H Rauhut, Compressive estimation of doubly selective channels in multicarrier systems: Leakage effects and sparsity-enhancing processing. IEEE J. Sel. Topics Signal Process. 4(2), 255271 (2010)

20. Universal Mobile Telecommunications System(UMTS): spatial channel model for Multiple Input Multiple Output(MIMO) simulations (3GPP, TS 36.900 Release 14) (2017), http://www.3gpp.org

21. A Liu, FB Zhu, VKN Lau, Close-loop autonomous pilot and compressive CSIT feedback resource adaption in multi-user FDD massive MIMO systems. IEEE Trans. Signal Process. 65(1), 173-183 (2017)

22. W Lu, Y Z Liu, D S Wang, 2011 2nd International Conference on Wireless VITAE, Compressed sensing in spatial MIMO channels (2011)

23. G Xu and H Liu, Proc. Int. Conf. Acoust. Speech Signal Process., An effective transmission beamforming scheme for frequency-division-duplex digital wireless communication systems (1995), 1729-1732

24. AF Molisch, A Kuchar, J Laaurila, et al., Geometry-based directional model for mobile radio channels principles and implementation. Eur. Trans. Telecommun. 14(4), 351-359 (2003)

25. M Ester, H P Kriegel, J Sander, X Xu, et al., Proceedings of the Second International Conference on Knowledge Discovery and Data Mining (KDD-96). A density-based algorithm for discovering clusters in large spatial data basis with noise (1996), 226-231

26. E Schubert, J Sander, M Ester, et al., DBSCAN revisited, revisited: why and how you should (still) use DBSCAN. ACM Trans. Database Syst. 42(3), 1-21 (2017)

27. W Dai, O Milenkovic, Subspace pursuit for compressive sensing signal reconstruction. IEEE Trans. Inf. Theory 55(5), 2230-2249 (2009)

28. R Chartrand, W Yin, Proceedings of 2008 IEEE International Conference on Acoustics, Speech and Signal Processing. Iteratively reweighted algorithms for compressed sensing (2008)

\section{Submit your manuscript to a SpringerOpen ${ }^{\circ}$ journal and benefit from:}

- Convenient online submission

- Rigorous peer review

- Open access: articles freely available online

- High visibility within the field

- Retaining the copyright to your article

Submit your next manuscript at $>$ springeropen.com 